\title{
Acupuntura na doença de Parkinson
}

\author{
Acupuncture in Parkinson's disease \\ Acupuntura en la enfermedad de Parkinson
}

\section{Resumo}

O estudo teve como objetivo caracterizar a produção científica sobre uso da acupuntura na Doença de Parkinson. Método: Revisão integrativa da literatura realizada em novembro de 2020, nas bases de dados LILACS, MEDLINE e PUBMED com os descritores: Acupuntura e Doença de Parkinson. Para seleção das produções foram critérios de inclusão: artigos publicados entre 2015 a 2020, nos idiomas português e inglês e de exclusão: editoriais, revisões de literatura, modelos animais e estudos duplicados. Resultados: Identificaram-se 29 artigos que, após leitura dos resumos e títulos e aplicação dos critérios de inclusão e exclusão culminou na seleção de quatro artigos como amostra deste estudo. Dentre os quatro artigos todos foram escritos em inglês, três eram estudos randomizados e um do tipo antes e depois; dois realizaram a intervenção durante oito semanas, um durante cinco e o outro durante três semanas. Os estudos abordaram o uso da acupuntura para as sintomatologias fadiga, distúrbios do sono e alterações motoras e os autores concluíram que a acupuntura é efetiva para melhorar a qualidade de vida dos pacientes com Doença de Parkinson. Conclusão: a acupuntura é viável de ser utilizada para amenizar sintomas do Parkinson. Contudo, constatou-se que o tema ainda é pouco explorado nas pesquisas e aponta necessidade de novos estudos para ampliar as evidências científicas sobre a efetividade do uso da acupuntura como prática não farmacológica no tratamento da Doença de Parkinson.

Palavras-chave: Acupuntura; Doença De Parkinson; Enfermagem.

\section{Abstract}

The study aimed to characterize the scientific production on the use of acupuncture in Parkinson's Disease. Method: Integrative literature review conducted in November 2020, in the LILACS, MEDLINE and PUBMED databases with the descriptors: Acupuncture and Parkinson's Disease. Inclusion criteria were selected for production: articles published between 2015 and 2020, in Portuguese and English and exclusion criteria: editorials, literature reviews, animal models 
and duplicate studies. Results: 29 articles were identified that, after reading the abstracts and titles and applying the inclusion and exclusion criteria, culminated in the selection of four articles as a sample of this study. Among the four articles, all were written in English, three were randomized studies and one was before and after; two performed the intervention for eight weeks, one for five and the other for three weeks. The studies addressed the use of acupuncture for symptoms of fatigue, sleep disorders and motor disorders and the authors concluded that acupuncture is effective in improving the quality of life of patients with Parkinson's disease. Conclusion: acupuncture is feasible to be used to alleviate Parkinson's symptoms. However, it was found that the topic is still little explored in research and points to the need for further studies to expand the scientific evidence on the effectiveness of using acupuncture as a nonpharmacological practice in the treatment of Parkinson's disease.

Keywords: Acupuncture; Parkinson Disease; Nursing.

\section{Resumen}

El estudio tuvo como objetivo caracterizar la producción científica sobre el uso de la acupuntura en la Enfermedad de Parkinson. Método: Revisión bibliográfica integradora realizada en noviembre de 2020, en las bases de datos LILACS, MEDLINE y PUBMED con los descriptores: Acupuntura y Enfermedad de Parkinson. Se seleccionaron criterios de inclusión para la producción: artículos publicados entre 2015 y 2020, en portugués e inglés y criterios de exclusión: editoriales, revisiones de la literatura, modelos animales y estudios duplicados. Resultados: se identificaron 29 artículos que, luego de leer los resúmenes y títulos y aplicar los criterios de inclusión y exclusión, culminaron en la selección de cuatro artículos como muestra de este estudio. Entre los cuatro artículos, todos fueron escritos en inglés, tres fueron estudios aleatorizados y uno fue antes y después; dos realizaron la intervención durante ocho semanas, uno durante cinco y el otro durante tres semanas. Los estudios abordaron el uso de la acupuntura para los síntomas de fatiga, trastornos del sueño y trastornos motores y los autores concluyeron que la acupuntura es eficaz para mejorar la calidad de vida de los pacientes con enfermedad de Parkinson. Conclusión: la acupuntura es factible para aliviar los síntomas de Parkinson. Sin embargo, se encontró que el tema aún está poco explorado en la investigación y apunta a la necesidad de más estudios para ampliar la evidencia científica sobre la efectividad del uso de la acupuntura como práctica no farmacológica en el tratamiento de la enfermedad de Parkinson.

Palabras clave: Acupuntura; Enfermedad de Parkinson; Enfermaría.

\section{Introdução}

A Doença de Parkinson (DP) é uma patologia caracterizada pela degeneração de neurônios, especialmente os dopaminérgicos, levando à disfunções na resposta motora, principalmente no tato fino, ocasionando na tétrade clássica: tremor em repouso, rigidez, bradicinesia e instabilidade postural (Fernandes \& Filho, 2018). Atinge ambos os sexos e aparenta maior frequência no sexo masculino. Os fatores predisponentes vão desde o estilo de vida, fatores ambientais e fator neuroprotetor do estrogênio (Carmo, 2015; Fernandes; Filho, 2018). O tratamento medicamentoso comumente utilizado é a Levodopa que possui efeitos adversos variados como alucinações, sonolência, hipotensão, náuseas; tratando a doença, mas não caminhando para o bem-estar do paciente (Giacomelli, 2018).

A acupuntura, uma das técnicas da medicina tradicional chinesa, visa estimular pontos no corpo na qual é possível restaurar o equilíbrio das energias no corpo para curar o paciente de suas doenças, promovendo o bem-estar e alívio de sintomas (YAEDU, 2011). A acupuntura é uma terapia complementar indicada e incentivada pela Organização Mundial da Saúde (OMS) que tem indicações da National Institutes of Health dos Estados Unidos para ser utilizada de forma isolada ou coadjuvante no tratamento de pós-quimioterapias, náuseas e vômitos, reabilitação após acidentes vasculares cerebrais, odontalgia pós-operatória, fibromialgia, osteoartrite, lombalgias, asma e etc (Espírito Santo et al, 2019).

No Brasil, a Portaria n ${ }^{\circ}$ 971/2006 aprovou a Política Nacional de Práticas Integrativas e Complementares (PNPIC) no Sistema Único de Saúde (SUS) e dentro dessas práticas estava a acupuntura.

O enfermeiro pode se especializar e praticar legalmente a acupuntura, e a prática segue as diretrizes da Política Nacional de Práticas Integrativas e Complementares do SUS inaugurada em 2006 seguindo os incentivos e recomendações da OMS (Cofen, 2018; Brasil, 2015). 
Conforme afirmado por Lopes, Nishiyama e Tanaka (2012), às drogas que são utilizadas no tratamento do Parkinson trazem alívios dos sintomas, porém levam a diversas reações adversas tais como: psicose, sedação, reações cardiovasculares, hipotensão ortostática, hipertensão arterial e sintomas gastrointestinais.

Neste contexto torna-se necessário o estudo aprofundado de práticas integrativas e complementares para o tratamento da Doença de Parkinson, prática esta que pode ser utilizada pelo enfermeiro (Cofen, 2018). Desta forma o presente estudo tem como objetivos caracterizar a produção científica sobre o uso de da acupuntura na Doença de Parkinson e descrever como esse tipo de terapia complementar pode ser utilizada nesta doença. Buscou-se quais eram as evidências científicas sobre o uso da acupuntura na Doença de Parkinson.

\section{Metodologia}

Trata-se de um estudo de Revisão Integrativa (RI) de natureza qualitativa que permite identificar, analisar e sintetizar estudos existentes e recentes sobre um determinado conhecimento possibilitando que o enfermeiro possa ter uma Prática Baseada em Evidências (PBE). Logo, trata-se de um método importante para analisar como está o nível das publicações, bem como reconhecer a variabilidade das mesmas. Portanto sintetizar os estudos existentes é de suma importância para área de saúde, pois tem o potencial de construir o conhecimento para tal área, produzindo então um saber fundamentado e uniforme para profissionais que nela atuam, para que possam realizar uma prática de qualidade (Pereira et al, 2018).

A produção de dados ocorreu em novembro de 2020 sendo desenvolvida nas seguintes etapas: $1^{\text {a }}$ : Elaboração da Questão norteadora: Quais as evidências científicas sobre o uso da acupuntura na Doença de Parkinson? 2a: Definição dos Critérios de inclusão: artigos publicados entre os anos de 2015 a 2020, disponíveis na íntegra online e gratuitos, nos idiomas português e inglês e, de exclusão: editoriais, revisões de literatura e estudos duplicados. $3^{\text {a }}$ : Definição dos descritores DECs Bireme: Acupuntura e Doença de Parkinson 4a: Busca nas Bases de Dados: BVS, Literatura Latino-Americana e do Caribe em Ciências da Saúde (LILACS), Medical Literature Analysis and Retrieval System online (MEDLINE); e na base de dados Public Medline (PUBMED). $5^{\text {a. }}$ Elaboração de Instrumento para coleta de dados. $6^{\text {a }}$ : Leitura e análise dos estudos incluídos na revisão integrativa e $7^{\mathrm{a}}$ etapa - Interpretação dos resultados.

\section{Resultados e Discussão}

Foram identificados 29 artigos que, após aplicação dos critérios de inclusão e exclusão (fluxograma 1) triagem dos títulos e resumos, culminou na seleção de quatro artigos para amostra do estudo, conforme o Fluxograma 1. 
Figura 1 - Fluxograma de seleção dos estudos.

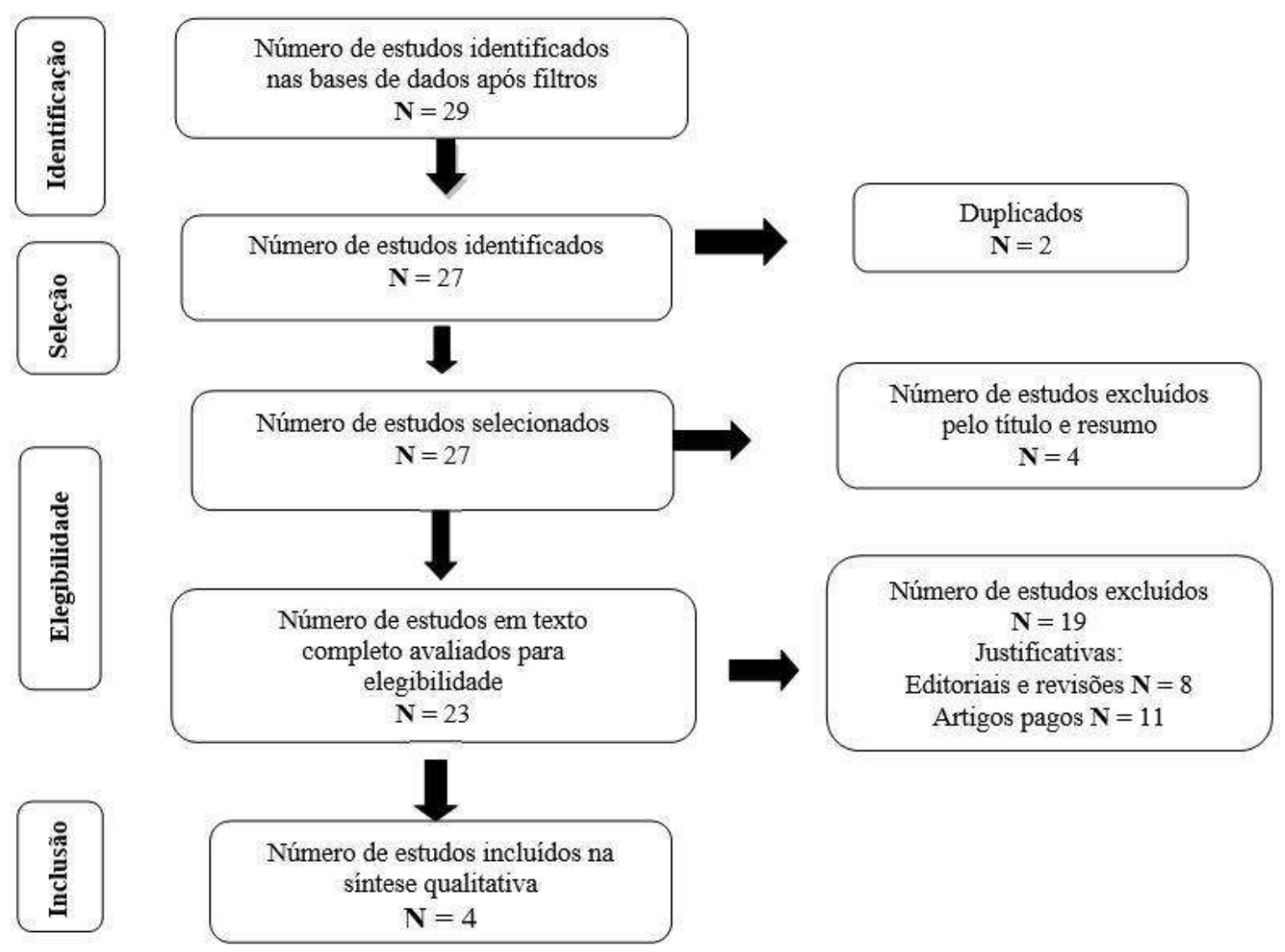

Fonte: Autores.

Dentre os quatro artigos selecionados, um foi publicado no Brasil, um em Singapura, um nos Estados Unidos e um na Coreia do Sul. Quanto à metodologia utilizada, três foram estudos clínicos randomizados e um do tipo antes e depois. Todos utilizaram escalas já existentes para avaliar o estado neurológico ou sintomas dos pacientes, sendo aplicadas as avaliações antes e depois do período de intervenção. Dos estudos analisados, três separaram os pacientes em grupo experimental e grupo controle para realizar comparações entre os grupos ao final do tempo de intervenção, e apenas um realizou a intervenção em todos os pacientes comparando apenas os resultados das escalas antes e depois da acupuntura. Quanto ao tempo de realização com acupuntura, dois estudos realizaram a intervenção durante oito semanas, um durante cinco semanas e o outro durante três semanas. (Quadro 1). 
Quadro 1 - Artigos selecionados para análise

\begin{tabular}{|c|c|c|c|c|}
\hline Estudo & Objetivo & Metodologia & Intervenção & Resultados \\
\hline $\begin{array}{l}\text { Aroxa et al. } \\
\qquad \begin{array}{c}(2017) \\
\text { Brasil }\end{array}\end{array}$ & \begin{tabular}{lr}
\multicolumn{2}{l}{ Avaliar os efeitos da } \\
acupuntura nos \\
distúrbios do & sono \\
em pacientes & com \\
DP. &
\end{tabular} & $\begin{array}{lr}\text { Estudo } & \text { clínico } \\
\text { randomizado } & \text { realizado } \\
\text { com 22 } & \text { pacientes, } \\
\text { avaliados } & \text { inicialmente } \\
\text { pelas escalas: } & \text { MEEM, HY } \\
\text { e PDSS; e reavaliação após } \\
8 \text { sessões. }\end{array}$ & $\begin{array}{l}\text { O grupo experimental } \\
\text { foi submetido a } 8 \\
\text { sessões semanais de } 30 \\
\text { minutos. }\end{array}$ & $\begin{array}{l}\text { Melhora da qualidade do sono } \\
\text { e psicose noturna, sintomas } \\
\text { motores noturnos, atividades } \\
\text { de vida diária, sonolência } \\
\text { diurna, fadiga e humor. }\end{array}$ \\
\hline $\begin{array}{l}\text { Yeo et al. } \\
\text { (2018) } \\
\text { Coréía do } \\
\quad \text { Sul. }\end{array}$ & $\begin{array}{l}\text { Examinar os efeitos } \\
\text { de } 8 \text { semanas de } \\
\text { acupuntura na } \\
\text { resposta cerebral e } \\
\text { nos sintomas da DP. }\end{array}$ & $\begin{array}{l}\text { Estudo comparativo } 1010 \\
\text { pacientes ambulatoriais } \\
\text { cujas respostas neurais e } \\
\text { comportamentais foram } \\
\text { avaliadas antes, durante e } \\
\text { após } 8 \text { semanas de } \\
\text { tratamento com } \\
\text { acupuntura utilizando as } \\
\text { escalas UPDRS, HY e } \\
\text { inventário de depressão de } \\
\text { Beck (BDI). }\end{array}$ & $\begin{array}{l}\text { Utilizada abordagem } \\
\text { semi-individualizada } \\
\text { em que os pacientes } \\
\text { foram tratados por } 15 \\
\text { minutos durante } 8 \\
\text { semanas rem } \\
\text { eletroacupuntura } r \\
\text { acupuntura manual } \\
\text { segundo sintomas } \\
\text { individuais do paciente. }\end{array}$ & $\begin{array}{llr}\text { Após } 8 \text { semanas } & \text { de } \\
\text { tratamento os escores } & \text { da } \\
\text { Escala Unificada } & \text { de } \\
\text { Classificação da Doença de } & \\
\text { Parkinson (UPDRS) e a } \\
\text { escala BDI diminuíram } \\
\text { estatisticamente } \\
\text { comparação aos escores antes } \\
\text { do tratamento, os escores } \\
\text { permanecerem estáveis } 8 \\
\text { semanas após a terapêutica. }\end{array}$ \\
\hline $\begin{array}{l}\text { Lei et al. } \\
\text { (2016) } \\
\text { Estados } \\
\text { Unidos. }\end{array}$ & $\begin{array}{l}\text { Avaliar eficácia da } \\
\text { eletroacupuntura } \\
\text { nos distúrbios da } \\
\text { marcha utilizando } \\
\text { tecnologia de } \\
\text { sensores em } \\
\text { pacientes com DP. }\end{array}$ & $\begin{array}{l}\text { Estudo randomizado } \\
\text { realizado com } 15 \\
\text { pacientes, por } 3 \text { semanas, } \\
\text { com sessões de } 30 \text { minutos } \\
\text { de acupuntura semanal }\end{array}$ & $\begin{array}{l}\text { Utilizado agulhas de } \\
\text { acupuntura e e étricos } \\
\text { estimuladores ére } \\
\text { na área motora sensorial } \\
\text { do pé, área de equilíbrio. }\end{array}$ & $\begin{array}{l}\text { Após } 3 \text { semanas de } \\
\text { acupuntura houve melhora da } \\
\text { marcha em pacientes do } \\
\text { grupo experimental }\end{array}$ \\
\hline $\begin{array}{l}\text { Kong et al. } \\
\text { (2017) } \\
\text { Singapura }\end{array}$ & $\begin{array}{l}\text { Avaliar a eficácia de } \\
5 \text { semanas de } \\
\text { acupuntura no } \\
\text { tratamento da fadiga } \\
\text { na DP }\end{array}$ & $\begin{array}{l}\text { Estudo randomizado com } \\
\text { avaliação dos pacientes } \\
\text { antes, após intervenção e } 4 \\
\text { semanas após a última } \\
\text { intervenção com uso das } \\
\text { escalas MFI, UPDRS, } \\
\text { PDQ-39, GDS. }\end{array}$ & $\begin{array}{l}\text { O tratamento foi } \\
\text { realizado } 2 \text { vezes na } \\
\text { semana com intervalos } \\
\text { de } 3 \text { dias por } 5 \text { semanas, } \\
\text { totalizando } 10 \text { sessões. }\end{array}$ & $\begin{array}{l}\text { A acupuntura real e simulada } \\
\text { é segura e eficaz na redução } \\
\text { da fadiga na DP. }\end{array}$ \\
\hline
\end{tabular}

Fonte: Autores.

Após análise dos resultados dos estudos observa-se que, em se tratando de uma doença que não possui cura, a acupuntura foi de alguma maneira eficaz no tratamento dos sintomas. Os estudos analisaram a terapia complementar e seus efeitos nos sintomas de fadiga, motores, gerais e distúrbios do sono, e em todas essas sintomatologias a acupuntura apresentou efeito benéfico ainda que não tenha aliviado por completo o sintoma estudado.

No estudo de Aroxa et al (2017), na coleta de dados após a realização do procedimento, os pacientes relataram melhora significativa da qualidade do sono noturno, psicose noturna, e sintomas motores noturnos, porém diversos outros sintomas não tiveram mudanças significativas como o início e manutenção do sono, atividade noturna, agitação, noctúria, sono relaxante e sonolência diurna. Observa-se que alguns sintomas bastante incômodos durante o sono não sofreram alterações; porém, no próprio estudo de Aroxa et al os participantes do estudo afirmaram que obtiveram melhora na qualidade do sono bem como no humor e na disposição para atividades de vida diária, o que consequentemente melhora a qualidade de vida.

Mendes e Barichello (2019, p. 3) afirmam que "qualidade de vida é um conceito abrangente afetado de uma maneira complexa pela saúde física, estado psicológico, nível de independência, relações sociais, crenças pessoais e características "ambientais". Assim, entende-se que qualidade de vida relacionada a saúde, a partir da perspectiva do sujeito, é um conceito complexo, subjetivo e pessoal que está relacionado a diversos aspectos do indivíduo que vão desde suas relações sociais, suas capacidades físicas, sua espiritualidade e etc. 
Os autores Sanches e Cardoso (2012) afirmam que as limitações físicas que incidem sobre os portadores da DP afetam emocionalmente os indivíduos pois não conseguem realizar suas atividades de vida diária como gostariam o que traz a eles a perda de sua independência, esse fato traz uma tendência ao isolamento social o que afeta diretamente a percepção destes indivíduos de sua qualidade de vida e de seu bem-estar emocional (Sanches; Cardoso, 2012).

Ao analisarmos os resultados da pesquisa do Aroxa et al (2017) percebe-se que, para os participantes do estudo, houve uma melhora da qualidade de vida. Os sintomas foram amenizados, o que melhorou a capacidade dos pacientes; este resultado pode gerar um grande impacto na vida destes indivíduos bem como no seu estado emocional, visto que a DP é uma doença neurodegenerativa e que a tendência é a progressão e agravamento dos sintomas.

No estudo de Yeo et al (2018) foi realizada uma avaliação dos pacientes com as escalas Escala Unificada de Avaliação para Doença de Parkinson UPDRS (avalia os sinais e sintomas e determinados por meio do autorrelato e da observação clínica), HY (compreende cinco estágios de classificação para avaliar a severidade da DP) e BDI (avalia sintomas depressivos). A avaliação foi feita antes, durante e depois do tratamento. Como resultado, observou-se uma melhora geral na escala UPDRS e a melhora na escala BDI, na qual permaneceram estáveis 8 semanas após o fim do tratamento; e não houve mudanças significativas na escala HY, ou seja, houve melhora nos sintomas da DP, porém não foi possível reduzir sua avaliação na escala HY. Os autores também realizaram exames de ressonância magnética na qual foi observado aumento da atividade neural na qual está intimamente ligado à melhora dos sintomas motores, chegando à conclusão de que a acupuntura teria a capacidade de neuromodular as áreas em repouso do cérebro.

Lei et al (2016) realizaram acupuntura em 15 pacientes na qual foram divididos em grupo experimental e grupo controle. No grupo experimental foi realizada eletroacupuntura e no controle foram utilizados pontos não acupunturais. Em ambos foi utilizada a mesma corrente elétrica. Após 3 semanas de tratamento houve uma melhora significativa no grupo experimental na maioria dos parâmetros de marcha medidos, especialmente na velocidade de marcha, comportamento, humor e atividades de vida diária, enquanto que no grupo controle não houve diferenças significativas.

No estudo de Kong et al (2017), os participantes foram divididos em dois grupos, um grupo iria receber sessões de acupuntura com o uso de agulhas reais e o outro grupo sessões de acupuntura utilizando agulhas falsas. $\mathrm{O}$ estudo teve como resultado a melhora dos sintomas de fadiga nos pacientes, porém os próprios autores chegam a conclusão e afirmam que os mecanismos da terapêutica são altamente placebo. Em contraponto podemos observar o estudo de Yeo et al (2018) na qual além dos resultados positivos das escalas aplicadas, ainda foi evidenciado através de exames de imagem a neuromodulação no sistema nervoso na qual explica as respostas positivas de melhora dos sintomas da doença.

Apesar da diferença de resultados obtidos entre os estudos de Kong et al (2017) e Yeo et al (2018), um demonstrando concretamente o mecanismo pela qual a terapêutica atua e o outro demonstrando resultados de efeito placebo, ambas as terapias demonstraram efeitos positivos nos pacientes.

Mediante os resultados positivos, seja pela intervenção ou pelo efeito placebo, frente a melhora na qualidade de vida relatada pelos participantes, entende-se que a acupuntura como terapia complementar é uma ferramenta importante para pessoas com Doença de Parkinson.

\section{Considerações Finais}

A acupuntura trouxe resultados favoráveis para amenizar diversos sintomas da DP com melhoria na qualidade de vida das pessoas com essa doença. Segundo os resultados dos estudos, o uso da acupuntura trouxe melhoras nos sintomas de fadiga, distúrbios do sono, alterações motoras e nos sintomas gerais da doença, além de melhorar a capacidade e disposição para a realização das atividades diárias, do humor e da percepção sobre a qualidade de vida dos participantes. 
Contudo, destaca-se como uma das limitações do estudo, a escassez de produções sobre o tema o que aponta a necessidade da realização de mais estudos sobre a efetividade da acupuntura aliada ao tratamento farmacológico da Doença de Parkinson.

As práticas integrativas e Complementares vêm avançando na sua utilização no campo da saúde abrindo outras possibilidades para a assistência às pessoas nos serviços de saúde, tendo como bases uma abordagem holística, que favorece o vínculo, a escuta ativa e o protagonismo do paciente.

Este estudo, embora com amostra limitada sobre DP e acupuntura, poderá trazer novas contribuições para o ensino, a pesquisa e a prática, na medida em que desperta a reflexão sobre a importância de práticas integrativas e complementares em pacientes com doenças crônicas. Contudo, entende-se ser necessário pesquisas mais amplas que utilizem abordagens distintas, com vista em fortalecer e disseminar o uso de técnicas e práticas alternativas.

Sugere-se então, para estudos futuros, realizar pesquisas utilizando escalas de qualidade de vida para tornar esse dado mais concreto, estudar a efetividade do uso da acupuntura no alívio das reações adversas da terapia farmacológica e utilizar mais, se possível, a ressonância magnética para evidenciar a neuromodulação do sistema nervoso.

\section{Referências}

Alvarez, A. M., Caldas, C. P., \& Gonçalves, L. H. T. (2019). PROENF Programa de Atualização em Enfermagem: Saúde do Idoso Ciclo 1, ARTMED.

Arouxa, F. H A., Gondim, I. T. G. O., Santos. E. L. W., Coriolano, M. G. W. S., Asano, A. G. C., \& Asano N. M. J. (2016). Acupuncture as Adjuvant Therapy for Sleep Disorders in Parkinson 's disease. J Acupunct Meridian Stud, 10 (1), 33-38. doi.org/10.1016/j.jams.2016.12.007

Brasil. Ministério da Saúde. (2015). Política Nacional de Práticas Integrativas e Complementares no SUS. Brasília. Diário Oficial da União. http://bvsms.saude.gov.br/bvs/publicacoes/politica_nacional_praticas_integrativas_complementares_2ed.pdf

Brasil. Ministério da Saúde. Portaria 971 de 3 de maio de 2006. Aprova a Política Nacional de Práticas Integrativas e Complementares (PNPIC) no Sistema Único de Saúde. Retrived from: http://bvsms.saude.gov.br/bvs/saudelegis/gm/2006/prt0971_03_05_2006.html

Carmo, M. R. S. (2015). Efeito neuroprotetor do antagonismo dos receptores p $2 x 7$ no parkinsonismo experimental induzido por 6 -ohda. Tese de doutorado, Universidade Federal do Ceará, Fortaleza, Brasil.

Espitiro Santo, F. H. et al. (2019) Práticas integrativas e complementares no cuidado ao idoso In Alvarez, A.M., Caldas, C.P., Gonçalves, L.H.T. Programa de Atualização em Enfermagem: Saúde do Idoso Ciclo 1(p.61-68), ARTMED.

Fernandes, I., \& Andrade Filho, A, S., (2018). Estudo clínico-epidemiológico de pacientes com doença de parkinson em salvador-bahia. Revista Brasileira de Neurologia e Psiquiatria, 22(1), 45-59. ESTUDO CLÍNICO-EPIDEMIOLÓGICO DE PACIENTES COM DOENÇA DE PARKINSON EM SALVADORBAHIA | Fernandes | Revista Brasileira De Neurologia E Psiquiatria (Revneuropsiq.Com.Br)

Giacomelli, A. O. (2018). Papel dos receptores purinérgicos em modelo animal de doença de Parkinson. Tese de doutorado, Universidade de São Paulo, São Paulo, SP, Brasil.

Kong, K. H., Ng, H. L., Ng, D. W., Tan, S. I, Tay, K. Y, Au, W. L., \& Tan. L. C. S. (2017). Acupuncture in thetreatmentof fatigue in Parkinson's disease: A pilot, randomized, controlled, study. Brain and behavior, 8(1), 1-6. https://doi.org/10.1002/brb3.897

Lei, H., Toosizadeh, N., Schwenk, M., Sherman, S., Karp, S., Stephenberg, E., \& Najafi, B. (2016). A Pilot Clinical Trial to Objectively Assess the Efficacy of Electroacupunctureon Gait in Patients with Parkinson's Disease Using Body Worn Sensors. PloSone, 11(5), 1-14. 10.1371/journal.pone.0155613

Lopes, M. A., Tanaka, E. M., \& Nishiyama, P. (2012). Práticas integrativas e complementares: Reações adversas causadas por antiparkinsonianoso cuidado ao idoso. Interfaces Científicas - Saúde e Ambiente, 1(1), 73-81. https://doi.org/10.17564/2316-3798.2012v1n1p73-81

Mendes, L. C, \& Barichello, E. (2019) Intervenções no manejo da fadiga e qualidade de vida em pacientes em quimioterapia: estudo de revisão. Cogitare enferm, 24, e61790. http://dx.doi.org/10.5380/ce.v24i0.61790.

Pereira, A. S. et al. (2018). Metodologia da pesquisa científica. UFSM. https://repositorio.ufsm.br/bitstream/handle/1/15824/Lic_Computacao_MetodologiaPesquisa-Cientifica.pdf?sequence $=1$.

Resolução COFEN 585/2018 de 8 de agosto de 2018. Estabelece e reconhece Acupuntura como especialidade e/ou qualificação profissional de Enfermagem. Retrived from: RESOLUÇÃO-585-2018-ACUPUNTURA.pdf (coren-ap.gov.br)

Sanches, K. C, \& Cardoso, K. G. (2012). Estudo da fadiga e qualidade de vida nos pacientes com doença de Parkinson. J Health SciInst, 30(4), 391-394. Retrived from: Estudo da fadiga e qualidade de vida nos pacientes com doença de Parkinson | J. Health Sci. Inst;30(4)

Souza, M. T. de, Silva, M. D. da, \& Carvalho, R. de. (2010). Revisão integrativa: o que é e como fazer. Einstein (São Paulo), 8(1), 102106. https://doi.org/10.1590/s1679-45082010rw1134 
Research, Society and Development, v. 10, n. 2, e45010212744, 2021

(CC BY 4.0) | ISSN 2525-3409 | DOI: http://dx.doi.org/10.33448/rsd-v10i2.12744

Yaedu, S. Y. (2011). O efeito da acupuntura, eletroacupuntura e estimulação nervosa elétrica transcutânea no tratamento dos sintomas de bradicinesia e hipocinesia na doença de Parkinson: uma nova perspectiva. Dissertação de mestrado. Universidade Federal do Paraná, Paraná, Brasil.

Yeo, S., van den Noot, M., Bosch, P., \& Lim, S. (2018). A study of the effects of 8-week acupuncture treatment on patients with Parkinson's disease. Medicine, 97 (50), 1-10. http://dx.doi.org/10.1097/MD.0000000000013434 